ARTICULOS ORIGINALES

Rev Chil Salud Pública 2020,

Vol 24(1): 23-29

\section{MAYORES CONCENTRACIONES DE CORTISOL, BIOMARCADOR DE ESTRÉS, EN RESIDENTES DE COMUNAS CON MENOR ÍNDICE DE CALIDAD DE VIDA URBANA}

\author{
HIGHER LEVELS OF CORTISOL, A BIOMARKER OF STRESS, AMONG \\ RESIDENTS OF MUNICIPALITIES WITH LOWER URBAN LIFE QUALITY \\ INDEX
}

\section{RESUMEN}

Introducción: Las desigualdades socioeconómicas y territoriales se relacionan con la salud de la población santiaguina; sin embargo, los mecanismos causales que generan las inequidades en salud no están del todo claros. En este trabajo, se cuantifica el estrés de individuos viviendo en diferentes comunas, y se lo discute como causa y consecuencia de procesos vinculados con inequidades en salud. El objetivo era relacionar el Índice de Calidad de Vida Urbana (ICVU) de la comuna de residencia de individuos en Santiago de Chile con las concentraciones de cortisol en pelo (CCP), un biomarcador de los niveles de estrés fisiológico crónico. Materiales y Métodos: Se exploró la relación entre CCP y la edad, el sexo y el ICVU de la Comuna de residencia en 80 estudiantes de educación superior en Santiago de Chile.

Resultados: No se encontraron correlaciones significativas entre CCP y la edad o el sexo de los participantes. Se encontró una correlación significativa entre CCP y los valores del ICVU de la comuna de residencia, además de encontrar diferencias significativas entre CCP y los distintos rangos de ICVU (Superior, Promedio e Inferior).

Discusión: Los resultados sugieren considerar las diferencias individuales de CCP como resultado de desigualdades socio-territoriales y también como posible causa de inequidades en salud. Se recomienda incluir el rol del estrés en las políticas públicas de salud, ampliando las redes de apoyo y prevención de estrés en los sectores con bajo ICVU.

Palabras claves: Segregación urbana, Cortisol en pelo, Estrés, Calidad de Vida, Inequidad en Salud

\begin{abstract}
Introduction: Socioeconomic and urban inequalities are associated with health in Santiago; nonetheless, the causal mechanisms underlying health inequalities are not entirely clear. This article quantifies the stress of individuals living in different municipalities and discusses its role as cause and consequence in the generation of health inequalities. The objective of this study was to determine the relationship between the Urban Quality of Life Index (UQoLI) of individuals' municipality of residence in Santiago, Chile with their hair cortisol concentrations (HCC), a biomarker of chronic physiological stress.

Materials and Methods: The relationship of HCC with age, sex and UQoLI was explored in a sample of 80 university students in Santiago, Chile.

Results: No significant correlations were found between HCC and age and sex. A significant correlation was found between HCC and UQOLI, and significant differences were found between HCC at different levels of UQoLI (High, Average, and Low).

Discussion: Results suggest that HCC differences could be considered a result of urban inequalities, as well as a possible cause of health inequalities. The role of stress should be included in public health policies, to expand support networks and prevent stress, especially among people living in areas with low urban quality of life.
\end{abstract}

Alejandro Díaz-Cavieres Facultad de Ciencias Sociales, Universidad de Chile, Santiago, Chile ale.diazcavieres@gmail.com

Hermann M. Niemeyer Facultad de Ciencias, Universidad de Chile, Santiago, Chile

Recibido el 24-06-2019 Aceptado el 12-12-2019
Key Words: Urban segregation, Hair cortisol, Stress, Quality of Life, Health inequalities 


\section{INTRODUCCIÓN}

Chile se posiciona como uno de los países más desiguales de la OCDE1 ${ }^{1}$, con diferencias socioeconómicas que obstaculizan el desarrollo nacional en diversos temas como educación, vivienda y salud². La desigualdad en Chile se manifiesta en una serie de condiciones estructurantes de la vida, tales como la escasez de recursos (e.g. ingresos, educación, vivienda, salud), inestabilidad (laboral, social), riesgo (seguridad financiera, social) y vulnerabilidad ${ }^{2}$. Estas características condicionan la vida de las personas influyendo en su disposición a actuar y su percepción del mundo $2,12,13$. Santiago, con niveles de desigualdad mayores que el promedio nacional ${ }^{3}$, reproduce estas condiciones sociales a través de una clara estructuración urbana según niveles socioeconómi$\cos ^{4}$.

Uno de los ámbitos que más resienten la desigualdad es la salud, entendida la salud como un estado de bienestar general que incluye, pero no se limita a la ausencia de patologías ${ }^{5}$. Si bien en las últimas décadas se ha producido una disminución general de problemas tales como la desnutrición, enfermedades infecciosas y mortalidad infantil, un menor nivel socioeconómico continúa estando asociado a una menor esperanza de vida ${ }^{6}$, mayor mortalidad infantil $^{7}$ y mayor prevalencia de enfermedades ${ }^{8}$ y de condiciones de riesgo tales como sobrepeso, malnutrición, alcoholismo, trastornos del sueño y sedentarismo ${ }^{8}$.

Posibles causantes de la inequidad (i.e. diferencias evitables e injustas) en salud son las diferencias en la calidad de las viviendas, el entorno social y el acceso a los servicios de salud ${ }^{9}$; no obstante, la extensión internacional del problema (con independencia de las diferencias en calidad de vivienda, servicios de salud, etc. $)^{5}$ y los resultados insatisfactorios que han tenido las políticas públicas 9,11 apuntan a la necesidad de comprender de mejor manera los mecanismos causales que relacionan desigualdad social con inequidad en salud ${ }^{9-11}$.

Un posible nexo entre desigualdad social e inequidad en salud es el nivel de estrés de las personas $^{13}$. En estudios internacionales, se ha detectado que residentes de barrios empobrecidos presentan mayores niveles de estrés ${ }^{10}$. Estas diferencias han sido explicadas tanto por la abundancia y el tipo de estímulos presentes como por la ausencia de mecanismos de defensa adecuados para su superación ${ }^{12,14,15}$.
El estrés, entendido como el proceso en el cual las demandas internas (i.e. percepciones, actitudes) y/o externas (i.e. pobreza, marginalidad) exceden la capacidad regulatoria del organismo ${ }^{13}$, se ha asociado a una serie de daños fisiológicos y/o psicológicos, afectando a muchas condiciones y patologías con mayor prevalencia en sectores de menor nivel socioeconómico $7,8,10$.

En consideración de la alta segmentación socioeconómica de Santiago de Chile ${ }^{4}$, surge la necesidad de explorar la relación entre el contexto socio-territorial y los niveles de estrés fisiológico crónico (i.e. concentración de cortisol en pelo: CCP) en residentes de distintos sectores de la capital.

Las hormonas indicadoras de estrés se liberan desde el sistema simpático-adrenal-medular (SAM) y el eje hipotalámico-hipofisiario-adrenal $(\mathrm{HHA})^{16}$. En conjunto, estos sistemas articulan una respuesta marcada en primera instancia (estrés agudo) por una liberación de catecolaminas (adrenalina, noradrenalina) desde el sistema SAM, seguida por glucocorticoides tales como el cortisol, provenientes del eje $\mathrm{HHA}^{15}$. La acumulación fisiológica de estas desregulaciones (respuestas agudas al estrés) se denomina estrés fisiológico crónico, y puede ser evaluado a través de la concentración de cortisol, ya sea en pelo ${ }^{17,18}$ sangre u orina ${ }^{18}$. La ventaja de la determinación en pelo radica en su crecimiento continuo, que genera un historial de concentración de cortisol a lo largo del tiempo ${ }^{18}$.

Por otra parte, es posible cuantificar la segmentación territorial de Santiago a través del Índice de Calidad de Vida Urbana ${ }^{19}$ (ICVU) de sus diferentes comunas, un indicador general de las condiciones de vida (vivienda, salud, educación, trabajo, conectividad) y del nivel socioeconómico de sus habitantes $^{19}$.

$\mathrm{Al}$ relacionar ambos indicadores, CCP e ICVU, se espera entregar una primera aproximación al rol del estrés como producto de la desigualdad socio-territorial y como productor de inequidades en salud.

\section{MATERIALES Y MÉTODOS}

El presente estudio se plantea como no experimental, transversal, cuantitativo y correlacional ${ }^{34}$. Los participantes fueron seleccionados sin asignación previa y categorizados posteriormente a partir de la comuna de residencia. La transversalidad indica que los datos fueron recogidos en un único momento ${ }^{34}$ y el diseño cuantitativo y correlacional busca esta- 
blecer relaciones estadísticas entre las variables, sin llegar a demostrar la dirección de causalidad en dichas relaciones.

Ochenta participantes fueron reclutados personal e individualmente durante enero de 2018. Los criterios de inclusión fueron tener entre 18 y 28 años de edad, estar inscritos en una institución de educación superior con sede en Santiago y tener una carga académica de 4 a 8 ramos o equivalente, incluyendo procesos de memoria y/o práctica profesional.

De acuerdo con la evidencia bibliográfica (condiciones que afectan el eje HHA ${ }^{13-16,18}$ ) y las necesidades metodológicas del estudio, se excluyeron a personas con pelo de una longitud inferior a $3 \mathrm{~cm}$ en la zona de la protuberancia occipital externa, mujeres embarazadas, personas con un índice de masa corporal $(\mathrm{IMC}=$ peso/altura2) mayor que 30 (obesidad clase I), consumidores de glucocorticoides sintéticos, deportistas de competición (más de $6 \mathrm{~h}$ semanales), trabajadores con más de $20 \mathrm{~h}$ por semana (o compromisos extracadémicos equivalentes, e.g. cuidado de enfermos, trabajos domésticos) y personas con las siguientes enfermedades diagnosticadas: síndrome de Cushing, enfermedad de Adisson, depresión, trastorno de ansiedad, trastorno bipolar, fatiga crónica, insomnio y alteraciones al eje hipotalámico-hipofisiario-adrenal (e.g. neoplasias, insuficiencia adrenal, etc.).

A cada participante se le tomó una muestra de $30 \mathrm{mg}$ de pelo recogidos de los $3 \mathrm{~cm}$ proximales en la zona adyacente a la protuberancia occipital externa20. A continuación, se le envió un cuestionario en línea a través del programa SurveyMonkey@, para que fuera respondido según la disponibilidad de tiempo de cada participante.

Todos los participantes firmaron el acuerdo de participación previamente aprobado por el Comité de Ética de la Investigación de la Facultad de Ciencias Sociales de la Universidad de Chile, siendo informados personalmente de todos los pormenores del estudio y de sus concentraciones personales de cortisol una vez finalizado el estudio.

Las muestras de pelo fueron analizadas utilizando la modificación de López-Barrales ${ }^{21}$ al protocolo de análisis de cortisol a partir de muestras de pelo propuesto por Davenport et al. ${ }^{22}$. Cada muestra fue lavada dos veces con $10 \mathrm{ml}$ de isopropanol utilizando un agitador (Finemixer SH2000, FinePCR, Seúl, Corea) por $3 \mathrm{~min}$ a $100 \mathrm{rpm}$ y posteriormente secada por exposición al ambiente, cortada en trozos de aproximadamente $1,5 \mathrm{~mm}$ de longitud y pulverizada en un agitador de bolas (Mini-Beadbeater-96; Biospec Inc., Baterlesville, OK, EEUU de NA) en dos instancias de 5 min cada una. Posteriormente, las muestras fueron pesadas, divididas en triplicados de alrededor de $10 \mathrm{mg}$ e incubadas en un agitador con $1 \mathrm{ml}$ de metanol a $50^{\circ} \mathrm{C}$ durante $16 \mathrm{~h}$ para la extracción del cortisol.

Los extractos fueron filtrados en pipetas Pasteur a través de lana de vidrio y luego reducidos a sequedad en un concentrador centrífugo (SVQ-120, Operon, Gyeonggi-do, Corea) por 1h. Al finalizar, se agregaron $100 \mu \mathrm{L}$ de PBS (phosphate buffered saline) de $\mathrm{pH}$ 8. La mezcla resultante fue analizada usando un kit comercial de inmunoensayo ELISA para muestras de cortisol en saliva (ELISA 11-CORHU-E01, ALPCO, Salem, NH, EEUU de NA) y luego leída en un lector de microplacas (EZRead 400, Biochrom, Cambridge, Reino Unido). Las densidades ópticas resultantes de la fotometría fueron transformadas a cantidad (ng) de cortisol por interpolación desde una regresión cuadrática de calibración obtenida a partir de las densidades ópticas de muestras estándares de concentración conocida de cortisol incluidas en el kit ELISA. Luego se determinó la concentración de cortisol ( $\mu \mathrm{g} / \mathrm{g}$ de pelo) de cada participante como el promedio entre los triplicados de cada muestra.

El cuestionario preguntó por el sexo, edad y domicilio mantenido por al menos 3 meses previos a la recolección de la muestra.

La comuna de residencia fue categorizada a partir del Índice de Calidad de Vida Urbana (ICVU) elaborado por el Instituto de Estudios Urbanos y Territoriales de la Pontificia Universidad Católica y la Cámara Chilena de la Construcción ${ }^{19}$. Este índice mide y compara en términos relativos la calidad de vida urbana a partir de la provisión e impacto socio-territorial de bienes y servicios, públicos y privados, en seis dimensiones: vivienda y entorno, salud y ambiente, condiciones socioculturales, ambiente de negocios, condición laboral y conectividad y movi$\operatorname{lidad}^{19}$. Además del índice particular para cada comuna, se establecen tres rangos, inferior, promedio y superior, de acuerdo con la ubicación relativa de cada comuna respecto del conjunto de ellas.

Se utilizó la prueba de Kruskal-Wallis para evaluar la relación entre CCP y sexo de los participantes y correlaciones de Spearman para evaluar la relación entre CCP y la edad de los participantes. La relación entre CCP e ICVU se examinó de dos maneras: mediante correlaciones de Spearman entre CCP y valores de ICVU y prueba de Kruskal-Wallis seguida 
de la prueba post hoc de Dunn para la relación entre CCP y rangos de ICVU (Superior, Promedio e Inferior)19. Los cálculos estadísticos fueron realizados con el programa Stata v.14.0.

\section{RESULTADOS}

La muestra estuvo constituida por 80 estudiantes (53 mujeres y 27 hombres), con una edad promedio de 22 años ( $\sigma=2,2$, mín.19, máx.28).
Se localizaron residencias en 21 comunas diferentes (tabla 1), con un ICVU promedio de 55 puntos ( $\sigma=10,4$; mín. 36,3 (Cerro Navia), máx. 71,4 (Providencia); Media para la Región Metropolitana $=46,6$; mín. 31,1 (La Pintana), máx. 71,4 (Providencia). La mayor concentración de participantes se estableció entre los rangos promedio y superior, quedando fuera de la muestra aquellas comunas con ICVU menores a 36,6 (Buin, Pedro Aguirre Cerda, Paine, Peñaflor, Talagante y La Pintana).

Tabla 1. Distribución de comunas de residencia presentes en la muestra, según rangos de ICVU19

\begin{tabular}{ll}
\hline \multicolumn{1}{c}{ Distribución por rangos del ICVU } & \multicolumn{1}{c}{ Comunas (de menor a mayor ICVU absoluto) } \\
\hline $\begin{array}{l}\text { Inferior (una desviación estándar o menos bajo el promedio nacional, } \\
\mathrm{n}=12 \text { ) }\end{array}$ & $\begin{array}{l}\text { Cerro Navia, San Bernardo, Conchalí, Puente Alto, Lo Prado, Indepen- } \\
\text { dencia, Huechuraba. }\end{array}$ \\
\hline Promedio (promedio nacional \pm una desviación estándar, n= 21) & $\begin{array}{l}\text { Quinta Normal, La Granja, Peñalolén, Estación Central, La Florida, } \\
\text { Maipú, Macul. }\end{array}$ \\
\hline $\begin{array}{l}\text { Superior (una desviación estándar o más sobre el promedio nacional, } \\
\mathrm{n}=46)\end{array}$ & $\begin{array}{l}\text { Santiago, Nuñoa, La Reina, San Miguel, Vitacura, Las Condes, Provi- } \\
\text { dencia. }\end{array}$ \\
\hline
\end{tabular}

Figura 1. Concentraciones de cortisol en pelo según rango de ICVU de la comuna de residencia $(n=80)$. La línea interna representa la mediana, los extremos de la caja los rangos intercuartiles y los puntos los valores atípicos.

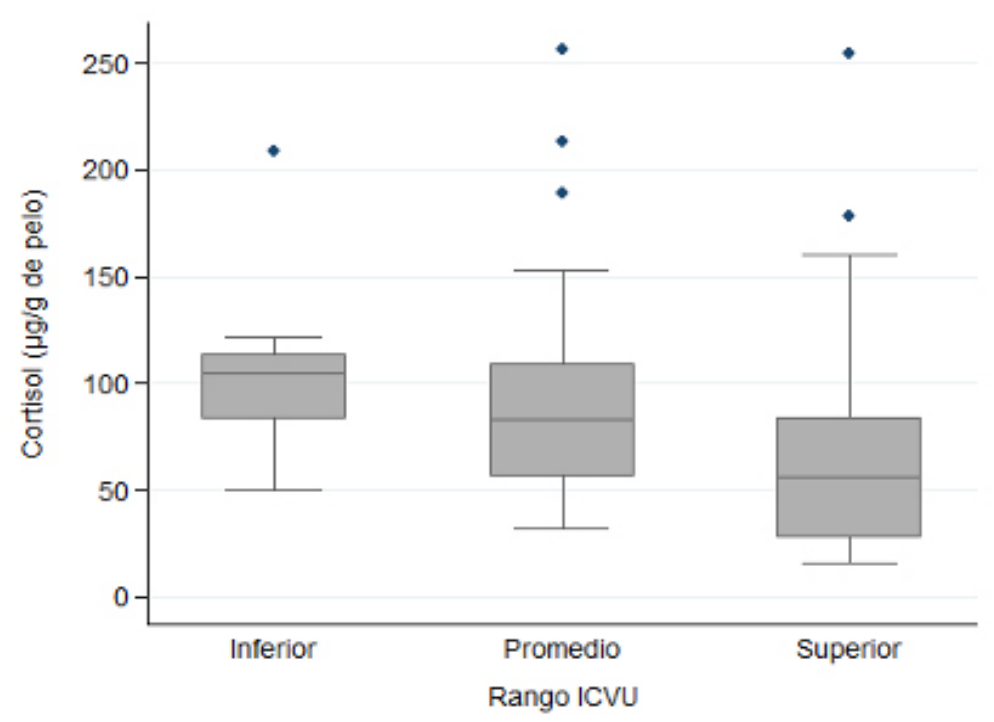


No se encontraron diferencias significativas en las CCP según sexo (prueba de Kruskal-Wallis: $\mathrm{H}=$ $0,515$; g.I. $=1 ; P=0,473)$ ni edad de los participantes (correlación de Spearman: $n=62 ; r=-0,123 ; p=$ 0,339).

Se encontró una correlación estadísticamente significativa entre CCP y valor del ICVU de la comuna de residencia (correlación de Spearman: $n=80 ; r=$ $-0,39 ; p=0,00039)$ y también diferencias significativas de las CCP entre los rangos de ICVU (prueba de Kruskal-Wallis: $n=80 ; H=12.137 ;$ g.I. $=2 ; p=0,002$ ). La prueba post-hoc de Dunn indicó que la diferencia entre los rangos Superior e Inferior del ICVU es altamente significativa $(Q=3,130 ; P=0,005)$, levemente no significativa entre Superior y Promedio $(\mathrm{Q}=2,288 ; \mathrm{P}=0,066)$ y altamente no significativa entre los rangos Promedio e Inferior $(\mathrm{Q}=1,175$; $\mathrm{P}=$ $0,720)$. También se encontró una diferencia significativa entre el rango Superior y el Promedio e Inferior agrupados (prueba de Kruskal-Wallis: $\mathrm{n}=80 ; \mathrm{H}=$ 10.758; g.l. $=1 ; p=0,001)$.

\section{DISCUSIÓN}

La relación establecida entre el ICVU de la comuna de residencia y las CCP de sus habitantes plantea una asociación entre el contexto socio-territorial y los niveles individuales de estrés fisiológico en una población estudiantil de Santiago. Considerando la multiplicidad de factores que inciden en la libera- ción de cortisol ${ }^{13,15,16}$ y la ausencia de relaciones con el sexo o la edad, la asociación encontrada plantea, por primera vez en Chile, la existencia de una segmentación socio-territorial de los niveles de estrés fisiológico en sus habitantes.

El carácter no experimental y correlacional de este estudio no permite concluir una relación de causalidad entre las variables examinadas; sin embargo, la evidencia ${ }^{10}$ apunta a que son las desigualdades urbanas las que se traducen en mayores o menores niveles de estrés según el ICVU de la comuna de residencia. Si bien más investigaciones son necesarias para establecer una causalidad que considere otras poblaciones, niveles socioeconómicos individuales y otras covariables, los resultados obtenidos permiten iniciar una discusión en pos de la inclusión del estrés en temas de inequidad en salud.

La diferenciación socio-territorial de los niveles individuales de estrés sugiere que los habitantes de comunas con menor ICVU están más expuestos a problemas gastrointestinales, cardiorespiratorios, de naturaleza inmune, metabólicos y cognitivos relacionados con mayores niveles de estrés ${ }^{16}$. La mayor frecuencia de muchos de estos problemas (obesidad, diabetes, problemas psicológicos) en sectores de menor nivel socioeconómico7,18 sugiere que el estrés podría ser a la vez consecuencia de la desigualdad socio-territorial y causa de las inequidades en salud. ${ }^{10,12}$ (figura 2).

El estrés como consecuencia de la desigualdad so-

Figura 2. Esquema del rol del estrés como consecuencia de la desigualdad y como posible causa de la inequidad en salud. Las líneas continuas representan relaciones probadas en la literatura y las punteadas aquellas relaciones con mayor incertidumbre.

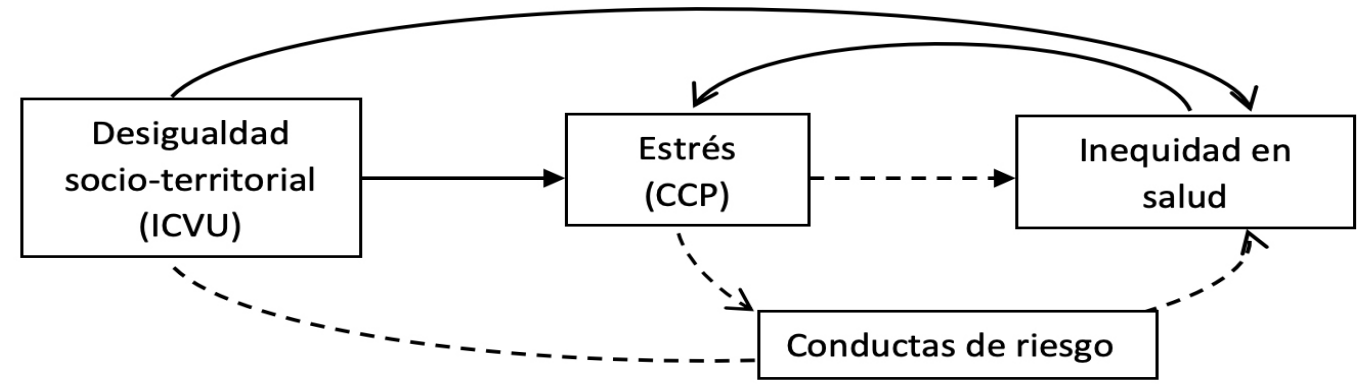


cio-territorial subraya la importancia de elementos contextuales como la conectividad, disponibilidad de áreas verdes, condiciones socioculturales, hacinamiento y seguridad ${ }^{19}$ en los niveles individuales de estrés fisiológico. Por otro lado, el rol del estrés como causa de la inequidad en salud contribuye a la comprensión de los mecanismos biológicos que subyacen la relación entre desigualdad social e inequidad en salud ${ }^{9,11}$.

Frente a esto, la incorporación del rol del estrés permite abordar la problemática de la inequidad en salud desde políticas de promoción, protección y restauración sanitaria que consideren la prevención y reducción de contextos estresores en comunas con menor ICVU.

Una oportunidad de mejora está en implementar políticas públicas y asignar recursos que permitan a los habitantes de sectores con menor ICVU prevenir y/o reducir sus niveles de estrés mediante el deporte, la cultura, las artes o el acompañamiento social y psicológico ${ }^{11}$. Al ampliar las redes de apoyo, es posible aumentar las estrategias de afrontamiento disponibles para los habitantes y de esta manera reducir la dependencia de conductas de riesgo como el consumo de grasas, tabaco o alcohol ${ }^{12}$ (figura 2). Un enfoque de este tipo permitiría, junto al aprovechamiento directo de los beneficiarios, impactar positivamente en los niveles de estrés de quienes, aún sin recibir beneficios directos, podrían mejorar su salud a partir de mejoras en su entorno comunitario (i.e. ICVU).

Junto a esto, aumentar las posibilidades de lidiar con el estrés de una manera constructiva aumenta también la posibilidad de prevenirlo, ya que al contar con una mayor red de apoyo y con mayores posibilidades de desarrollo, la persona beneficiada de estas políticas públicas puede reducir su percepción de vulnerabilidad ante el ambiente ${ }^{14,15}$, y contar con un mayor número de herramientas para relacionarse con las dificultades diarias.

En conclusión, junto a la necesidad de robustecer la investigación en torno al estrés fisiológico en Chile, este trabajo espera aportar a la comprensión y la discusión del rol que tiene el contexto socio-territorial en los cuerpos y mentes de los habitantes de Santiago.

\section{Agradecimientos}

Se agradece la valiosa ayuda del personal colaborador en el Laboratorio de Química Ecológica de la Facultad de Ciencias de la Universidad de Chile.

\section{Conflictos de interés}

Los autores declaran no tener conflictos de intereses.

\section{REFERENCIAS BIBLIOGRÁFICAS}

1. Organization for Economic Co-operation and development. OECD. Society at a glance: OECD Social Indicators. 5ta Ed.: OECD Publishing. Paris; 2016.

2. Programa de las Naciones Unidas para el Desarrollo. Desiguales. Orígenes, cambios y desafíos de la brecha social en Chile [en línea]. Santiago: Uqbar Editores; 2017 [consultado en mayo 2019]. Disponible en: http://www.cl.undp.org/content/dam/chile/docs/pobreza/undp_cl_pobreza-Libro-DESIGUALES-final.pdf

3. Agostini C, Hojman D, Valenzuela L. Segregación residencial de ingresos en el Gran Santiago, 1992-2002: una estimación robusta. EURE [en línea]. 2016 [consultado en marzo de 2019];42(127):159-184. http:// www.eure.cl/index.php/eure/article/view/1732/920

4. Agostini C. Pobreza, desigualdad y segregación en la región metropolitana [en línea]. CEP. 2010; [consultado en marzo de 2019] 117. Disponible en: https:// www.cepchile.cl/cep/site/artic/20160304/asocfile/20160304095213/rev117_agostini.pdf

5. World Health Organization (WHO). Towards a Conceptual Framework for Analysis and Action on the Social Determinants of Health [en línea]; 2008 [consultado en marzo de 2019]. Commission on the Social Determinants of Health (CSDH). Disponible en: http:// www.who.int/social_determinants/en/

6. Sánchez H, Albala C. Desigualdades en salud: adulto en comunas del Gran Santiago. Rev Méd Chile [en línea]. 2004 [consultado en abril de 2019];132(4):453-460. Disponible en: https:// scielo.conicyt.cl/scielo.php?script=sci_arttext\&pi$\mathrm{d}=$ S0034-98872004000400007\&lng=es

7. Donoso E. Desigualdades en mortalidad infantil entre las comunas de la provincia de Santiago. Rev Méd Chile [en línea]. 2004 [consultado en marzo de 2019];132(4): 461-466. Disponible en: https://dx.doi. org/10.4067/S0034-98872004000400008

8. Chile. Ministerio de Salud. Encuesta nacional de salud ENS Chile 2009-2010 [en línea]. Chile: MINSAL; Facultad de Medicina de la Pontificia Universidad Católica de Chile; Universidad Alberto Hurtado; 2010 [consultado en junio de 2019]. Disponible en: https:// www.minsal.cl/portal/url/item/bcb03d7bc28b64dfe040010165012d23.pdf 
9. Chile. Ministerio de Salud. Estrategia Nacional de Salud para el cumplimiento de los Objetivos Sanitarios de la década 2010-2020 [en línea]. Santiago: MINSAL; 2011 [consultado en mayo de 2019]. Disponible en: https://www.minsal.cl/portal/url/item/c4034eddbc96ca6de0400101640159b8.pdf

10. Cohen S, Doyle WJ, Baum A. Socioeconomic Status is associated with stress hormones. 2006;68(3):414-420.

11. Whitehead M, Jennie P. Swimming upstream? Taking action on the social determinants of health inequalities. Soc Sci Med. 2010;71(7):1234-1236.

12. Holst M, Ekholm O, Nielsen L, Kjær A, Kronborg C, Tanggard P. Associations between perceived stress, socioeconomic status, and health-risk behaviour in deprived neighbourhoods in Denmark: a cross-sectional study. BMC Public Health [en línea]. 2018 [consultado en junio 2019];18:250. Disponible en: https:// www.ncbi.nlm.nih.gov/pmc/articles/PMC5812195/ pdf/12889_2018_Article_5170.pdf

13. Ice G, James G. Measuring stress in humans. A practical guide for the field. Cambridge University Press; 2007.

14. Miller G, Chen E, Zhou E. If it goes up, must it come down? Chronic stress and the hypothalamic-pituitary-adrenocortical axis in humans. Psychol Bull. 2007;133(1):25-45.

15. Koolhaas J, De Boer S, Coppens C, Buwalda B. Neuroendocrinology of coping styles: towards understanding the biology of individual variation. Front Neuroendocrinol. 2010;31(3):307- 321.

16. Chrousos G. Stress and disorders of the stress system.
Nat Rev Endocrinol. 2009; 5(7):374-381.

17. Raul J, Cirimele V, Ludes B, Kintz P. Detection of physiological concentrations of cortisol and cortisone in human hair. Clin Biochem. 2004;37(12):1105-1111.

18. Stalder T, Kirschbaum C. Analysis of cortisol in hair - State of the art and future directions. Brain Behav Immun. 2012;26(7):1019 1029.

19. Pontificia Universidad Católica de Chile; Cámara Chilena de la Construcción. Índice de Calidad de Vida Urbana: comunas y ciudades de Chile [en línea]. Instituto de Estudios Erbanos y Territoriales; 2018 [consultado en mayo 2019]. Disponible en: http://estudiosurbanos.uc.cl/images/investigaciones/Arturo_Orellana/ ICVU_2018/20180508_ICVU_2018_-_Version_Definitiva.pdf

20. XieQ, GaoW, Li J, QiaoT, Jin J, Deng H, Lu Z. Correlation of cortisol in 1 -cm hair segment with salivary cortisol in human: hair cortisol as an endogenous biomarker. Clin Chem Lab Med. 2011;49:2013-2019.

21. López R. Estrés biomecánico y fisiológico en poblaciones prehispánicas de San Pedro de Atacama [en línea]. Santiago, Chile: Universidad de Chile, Facultad de Ciencias Sociales; 2013 [consultado en junio 2019]. Disponible en: http://repositorio.uchile.cl/ handle/2250/131261

22. Davenport D, Tiefenbacher S, Lutz C, Novak M, Meyer J. Analysis of endogenous cortisol concentrations in the hair of rhesus macaques. General and comparative endocrinology. 2006;147(3): 255-261. 